\title{
Molecular Study of Cutaneous Leishmaniasis Human Reservoirs and Infections in Bastak
}

\author{
Mohammad Palangar, Houshang Jamali* and Atefahekhosraubak \\ ${ }^{1}$ Department of Microbiology, Islamic Azad University, Iran \\ *Corresponding author: Houshang Jamali, Department of Microbiology, Jahrom Branch, Islamic Azad University, Jahrom, Iran
}

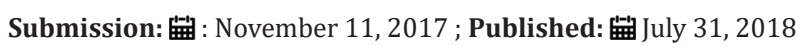

\begin{abstract}
Introduction: Leishmaniasis is one the six most important diseases in tropical areas. Study and research on various aspects of it is recommended and supported by the World Health Organization. Broom tail rodents (Rodentia: Muridae: Gerbillinae) are the most important reservoir host of parasite causes wet cutaneous leishmaniasis (or Zoonotic Cutaneous Leishmaniasis (ZCL) disease) means leishmania major. Different species of rodents from different regions of Iran play role in maintenance of the parasite. The purpose of this research is molecular study of cutaneous leishmaniasis human reservoirs and infections in Bastak County, Hormozgan Province, Iran.
\end{abstract}

Materials and methods: Live traps were used to catch rodents. The rodents were anesthetized with chloroform in the field and four slides were prepared from earlobe of each animal by sanding method and their morphometric characteristics were completely measured and recorded. Rodent abdomen was opened with blade and the liver and the spleen of each sample were kept in a numbered container containing $70 \%$ ethanol. The prepared slides were examined with microscopic (Giemsa staining) and molecular methods. Finally, using Nested-PCR method, they were evaluated using species-specific primers (LIN R4, LIN 17, LIN 19).

Result: A total of 108 rodents from five species of three different genera were collected. Merionespersicus species with $26.9 \%$ was the predominant species. A contamination in T. indica species and two cases of contamination in two samples of female M. hurrianae were observed. L. major species was also identified.

Conclusion: Broom tail rodents are abundance and have relatively high diversity in Bastak County. They live in proximity to human dwellings. Tateraindica and Merioneshurrianae species are introduced as the disease reservoir hosts in this area and use of molecular and PCR methods provides the possibility of faster diagnosis of parasite species.

Keywords: Cutaneous leishmaniasis; Nested-PCR; Tateraindica; Merioneshurrianae. L. major

\section{Introduction}

Leishmaniasis (Cutaneous leishmaniasis (CL)) has long been known in Iran. In Iranian ancient books, including Canon of Medicine compiled by Avicenna (Ibn Sina), a wound called "khyroniyeh" was mentioned. Its treatment was difficult and lasted a long time and was resistant to different medications. By referring to signs and its symptoms mentioned about the wound, it is thought to be lesion of leishmaniasis [1]. Cutaneous leishmaniasis is a chronic disease of skin and named differently in various areas (e.g., it is known to Salak in Shiraz and Lakehye Sal in Mashhad). The disease is especially observed in warm seasons and in tropical regions. It is caused by a protozoan of genus leishmania and Trypanosomatidae family. It can be transferred between human and some animals, including rodents and dogs and it can also transfer by a sandfly (Phlebtomus) from a patient to others. Leishmaniasis arises at first as a small papule and then becomes larger (nodule), and finally converts to wound. It is possible that the wounds recover spontaneously in a few weeks or even several years [2].
Leishmaniasis is one of the most common parasitic diseases in Iran that is called urban (dry) and rural (wet) Leishmaniasis. Leishmaniatropica and L. major species are agents of urban (dry) leishmaniasis and rural (wet) leishmaniasis, respectively [3]. The presence of parasite can be identified by microscopic observation of slides stained with Giemsa. However, its type is not recognizable [4]. Molecular techniques are widespread in recent years to detect leishmaniasis. One of these techniques is determination of parasite type by PCR method. This method can be done even with small number of parasites in a sample [5]. Leishmaniasis is not so problematic in comparison with other diseases. However, the wounds caused by it due to various reasons may leave some traces as improper and disfiguring scars. Identification of parasite species is effective in adoption of the disease prevention and control programs. Its classification to different species and strains is very difficult due to several reasons and similarity in appearance of parasite species. Epidemiological and clinical evidence are not 
alone effective in differentiating between various species [6]. Since the first step in planning for control and fighting a disease is determination of exact specification of pathogens, molecular studies that in addition to detection of genetic diversity among species and within species, diagnose the genus of Leishmania, are of great importance $[7,8]$.

So, study and investigation of cutaneous leishmaniasis molecular reservoirs and human infections is necessary to control the parasite. Since the evaluation process of the disease is greater in warm climates, Bastak County with a warm climate is a suitable area to evaluate the subjects.

\section{Methods}

To determine reservoirs of cutaneous leishmaniasis and parasites cause the disease in the patient, rodents' hunting was performed in spring, autumn and winter of 2014 in selected villages of Bastak County (the city of Bastak location; longitude: 54 degrees and 23 minutes; Latitude: 27 degrees and 14 minutes; height 2120 $\mathrm{m}$ above sea level), which were selected from different climatic regions of the County. Once in a month and each time 10 Sherman live traps were installed at sun sets around active nests and they were collected in morning of the next day. Date palm, cucumber and walnuts were used as bait.

After collecting the traps containing rodent, they were placed in a plastic bag and were anesthetized with chloroform-soaked cotton. When the researchers became sure of the animal death, its abdomen was opened using a sterile surgical blade and the liver and spleen were placed in a numbered container containing $70 \%$ ethanol. After clearing the abdomens, rodent bodies in containers containing $10 \%$ formalin were sent for taxidermy and identification to Zoology Laboratory of Shiraz University. Rodents' identification was conducted in Zoology Laboratory of Natural History Museum of Shiraz University and by the help of Iranian rodent diagnostic key [1]. To confirm the suspicious samples, they were sent to Rodent Research Center of Mashhad University.

Two slides were prepared from Serosity of the ear and the tail of each animal using sanding method. The prepared slides from the ears and the tails of the rodents were examined using microscopic method and Giemsa staining for observation of amastigotes. In case of observation of Leishman body, DNA extraction process began for the positive slides using Motazedian et al. [9] method. DNA extraction from the liver and the spleen was also carried out using

\section{Result}

Table 1: Frequency and percentage of rodent hunting in the villages studied in Bastak County.

\begin{tabular}{|c|c|c|c|c|c|c|c|}
\hline \multirow{2}{*}{ Location Species } & \multicolumn{2}{|c|}{ Kukherd } & \multicolumn{2}{c|}{ Gachuyeh } & \multicolumn{2}{c|}{ Hanguyeh } & \multicolumn{2}{c|}{ Bastak } \\
\cline { 2 - 9 } & Frequency & $\%$ & Frequency & $\%$ & Frequency & Frequency & \% \\
\hline Merionespersicus & 0 & $0 \%$ & 0 & $0 \%$ & 1 & $0.9 \%$ & 0 \\
\hline Tateraindica & 0 & $0 \%$ & 0 & $0 \%$ & 0 & $0 \%$ & 2 \\
\hline Merioneshurrianae & 4 & $3.7 \%$ & 9 & $8.3 \%$ & 11 & $10.2 \%$ & 0 \\
\hline Merioneslybicus & 0 & $0 \%$ & 1 & $0.9 \%$ & 0 & $0 \%$ & $0 \%$ \\
\hline
\end{tabular}

Azizi et al. [10-12] method. Nested-PCR-based approach was used for proliferation of variable region of Leishmania kinetoplast DNA (k DNA) minicircle. LIN R4, LIN 17 and LIN 19 were used in this approach as a forward primer in both stages, reverse primer of the first stage and reverse primer of the second stage, respectively.

A. - -LIN R4: 5'- GGG GTT GGT GTA AAA TAGGG-3'.

B. - -LIN 17: 5'- TTT GAA CGG GAT TTC TG-3'.

C. -LIN 19: 5' - CAG AAC GCC CCT ACC CG-3'.

This technique was designed in 2000 by Aransay et al. [4] and it was used by the author in numerous studies for diagnosis of Leishmania parasite in carriers' body and Leishmania reservoirs [13].

The reference strains of the parasite were used as follows to set up PCR reaction: the strains

Infantum: MCAN/IR/96/Lon49Le.

Le.Tropica: MHOM/IR/89/ARD2

Major: MHOM/IR/54/LV39Le.

The above strains were used for comparison. These strains were taken from Department of Parasitology and Mycology, School of Medicine, Shiraz University of Medical Sciences. Negative control, which was the extracted DNA from the liver of healthy rodents, was used for all reactions to ensure of the accuracy of performance. Thermal profile at the first stage was as follows: after a preliminary heat at $94^{\circ} \mathrm{C}$ for $5 \mathrm{~min}$ (Initial Denaturation), proliferation continued for 30 seconds at $94^{\circ} \mathrm{C}$ (Denaturation), 30 seconds at $52^{\circ} \mathrm{C}$ (Annealing) and 1 minute at $72^{\circ} \mathrm{C}$ (Extension) and finally, 10 minutes at $72^{\circ} \mathrm{C}$ (Final Extension) (the first stage was repeated in 30 cycles). The second phase of thermal profile was also similar to the first stage, but without preliminary heat and the heat relatedto Annealing stage was $58^{\circ} \mathrm{C}$. The second thermal profile was repeated 33 cycles.

After proliferation of DNA, 5 microliter of PCR product mixed with loading buffer was electrophoresed for $40 \mathrm{~min}$ on $1.5 \%$ agarose gel containing ethidium bromide. By detection in UV Transilluminator device, the obtained bonds from the samples were compared with each other and studied. They were also compared with the obtained bonds from the standard strains and were recognized. The results were analyzed using SPSS statistics 19 and Post Hoc test, Anova, paired t-test and chi-square tests. 


\begin{tabular}{|c|c|c|c|c|c|c|c|c|}
\hline Gerbillusnanus & 3 & $2.8 \%$ & 6 & $5.6 \%$ & 8 & $7.4 \%$ & 0 & $0 \%$ \\
\hline Total & 7 & $6.5 \%$ & 16 & $14.8 \%$ & 20 & $18.5 \%$ & 2 & $1.9 \%$ \\
\hline
\end{tabular}

108 rodents of 5 different species were caught in this study. Rodents' hunting was done completely out of rural places and borders and as a result, no house mice (Mus Musculus) commonly hunted in the studies for determination of rodent fauna, were caught. Species, frequency and percentage of rodent hunting in the studied areas of Bastak County are listed in (Table 1) and (Table 2). Frequency percentage of species in the city of Kukherd showed that the highest frequencies with $57.1 \%$ and $42.9 \%$ was related to Merioneshurrianae and Gerbillusnanus species, respectively (Table 1). Frequency percentage of species in Gachuyeh Village showed that the highest frequencies with $56.3 \%, 37.5 \%$ and $6.3 \%$ were related to Merioneshurrianae and Gerbillusnanus and Merioneslybicus species, respectively (Table 1). Frequency percentage of species in Hanguyeh Village showed that the highest frequencies with $55 \%$, $40 \%$ and $5 \%$ were related to Merioneshurrianae and Gerbillusnanus

and Merionespersicus species, respectively (Table 1). Frequency percentage of species in the city of Bastak showed that the highest frequency with $100 \%$ was related to Tateraindica species (Table 1).

Frequency percentage of species in Guwdah Village showed that the highest frequencies with 50\%, 27.8\%, 11.1\% and $11.1 \%$ were related to Merionespersicus, Tateraindica, Merioneshurrianae and Merioneslybicus species, respectively (Table 2). Frequency percentage of species in Deh Tall Village showed that the highest frequencies with $36.4 \%, 36.4 \%$ and $27.3 \%$ were related to Merionespersicus, Tateraindica and Merioneslybicus species, respectively (Table 2). Frequency percentage of species in Dehang Village showed that the highest frequencies with $47.8 \%$ and $52.2 \%$ were related to Merionespersicus and Tateraindica species, respectively (Table 2).

Table 2: Frequency and percentage of rodent hunting in the villages studied in Bastak County.

\begin{tabular}{|c|c|c|c|c|c|c|c|c|}
\hline \multirow{2}{*}{ Location Species } & \multicolumn{2}{|c|}{ Guwdah } & \multicolumn{2}{c|}{ Deh Tall } & \multicolumn{2}{c|}{ Dehang } & \multicolumn{2}{c|}{ Total } \\
\cline { 2 - 9 } & Frequency & $\mathbf{\%}$ & Frequency & $\%$ & Frequency & \% & Frequency & \% \\
\hline Merionespersicus & 9 & $8.3 \%$ & 8 & $7.4 \%$ & 9 & $8.3 \%$ & $7.4 \%$ \\
\hline Tateraindica & 5 & $4.6 \%$ & 8 & $7.4 \%$ & 5 & $4.6 \%$ & 8 & $7.4 \%$ \\
\hline Merioneshurrianae & 2 & $1.9 \%$ & 0 & $0 \%$ & 2 & $1.9 \%$ & 0 & $0 \%$ \\
\hline Merioneslybicus & 2 & $1.9 \%$ & 6 & $5.6 \%$ & 2 & $1.9 \%$ & 6 & $5.6 \%$ \\
\hline Gerbillusnanus & 0 & $0 \%$ & 0 & $0 \%$ & 0 & $0 \%$ & 0 \\
\hline Total & 18 & $16.7 \%$ & 22 & $20.4 \%$ & 18 & $16.70 \%$ & 22 & $20.4 \%$ \\
\hline
\end{tabular}

Using Fisher's Exact Test, it was found that there was a significant relationship at level of $1 \%$ between studied location and species (P-Value=0.000).

Sampling process using microscopic method was done from all hunted rodents to recognize Leishmania contamination. They were also studied in terms of DNA detection of Leishmania parasite in their liver and spleen. The results are separately specified in (Table 3). Using Fisher's Exact Test, it was found that there was no significant relationship between contamination and species (P-Value=0.707). The approved contamination in all samples was related to female Tateraindica and female Merioneshurrianae species (Figure 1).

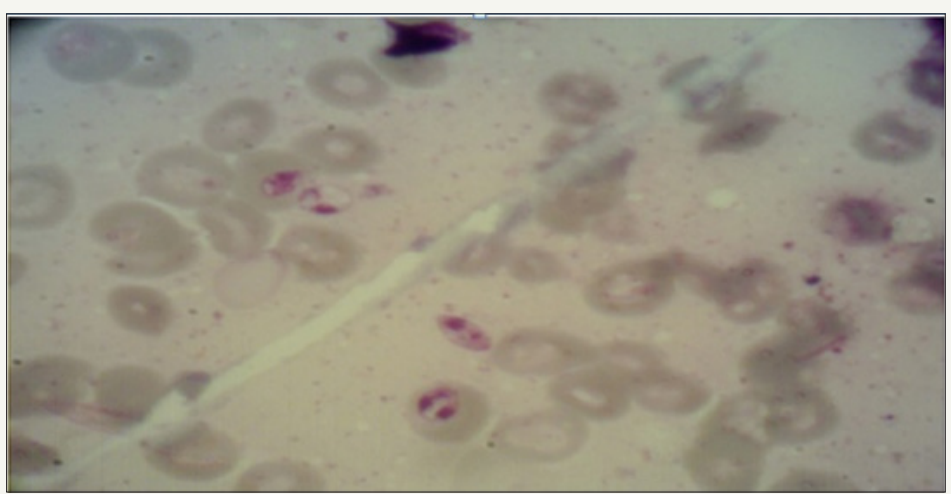

Figure 1: Amastigote of Leishmaniasis major in prepared sample from Meriones hurricane species, Bastak County.

Table 3: Results related to identification of Leishmania reservoirs.

\begin{tabular}{|c|c|c|c|c|c|c|c|c|}
\hline \multirow{2}{*}{ Contamination Species } & \multicolumn{2}{|c|}{ Microscopic } & \multicolumn{2}{c|}{ PCR } & \multicolumn{2}{c|}{ Non } & \multicolumn{2}{c|}{ Total } \\
\cline { 2 - 9 } & Frequency & $\mathbf{\%}$ & Frequency & $\mathbf{\%}$ & Frequency & $\%$ & Frequency & \% \\
\hline Merionespersicus & 0 & $0 \%$ & 0 & $0 \%$ & 29 & $26.9 \%$ & 29 & $26.9 \%$ \\
\hline
\end{tabular}




\begin{tabular}{|c|c|c|c|c|c|c|c|c|}
\hline Tateraindica & $1(\%)$ & $0.9 \%$ & $1(\%)$ & $0.9 \%$ & 25 & $23.1 \%$ & 27 & $25.0 \%$ \\
\hline Merioneshurrianae & $1(\%)$ & $0.9 \%$ & $2(\%)$ & $1.9 \%$ & 23 & $21.3 \%$ & 26 & $24.1 \%$ \\
\hline Merioneslybicus & 0 & $0 \%$ & 0 & $0 \%$ & 9 & $8.30 \%$ & 9 & $8.3 \%$ \\
\hline Gerbillusnanus & 0 & $0 \%$ & 0 & $0 \%$ & 17 & $15.7 \%$ & 17 & $15.7 \%$ \\
\hline Total & 2 & $1.9 \%$ & 3 & $2.8 \%$ & 103 & $95.4 \%$ & 108 & $100.00 \%$ \\
\hline
\end{tabular}

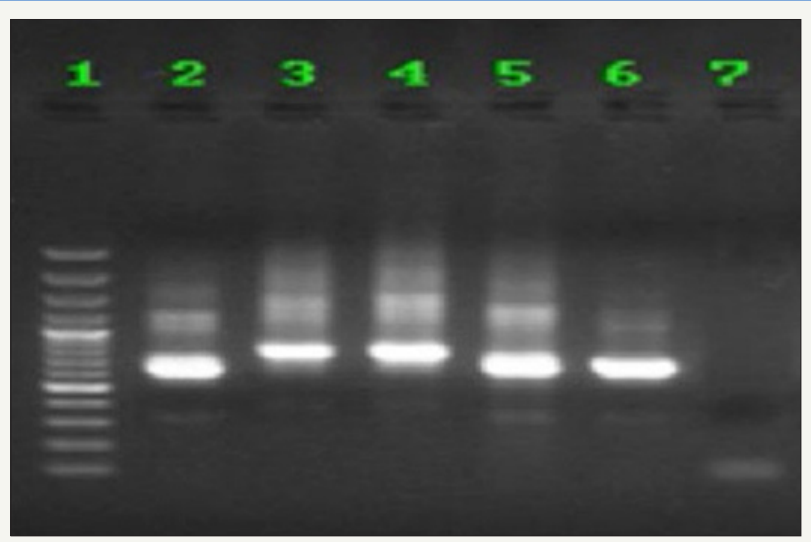

Figure 2: The results of electrophoresis of Nested-PCR products related to rodent samples of Bastak County, Hormozgan Province, Iran, with LIN R4, LIN R17 and LIN 19 primers in 1.5\% agarose gel; size marker (column 1); L. major standard strain (column 2); Leishmaniatropica standard strain (column 3); Leishmaniainfantum standard strain (column 4); a Merioneshurrianae sample (column 5); a Tateraindica sample (column 6); negative control (column 7).

The results of Nested-PCR: determined genes by LIN R17, LIN R4 and LIN 19 primers on agarose gel using Nested-PCR are shown in (Figure 2).

\section{Discussion}

Five species of rodents (Gerbillinae subfamily) were identified as broom tail rodents in this study in Bastak County. The highest frequencies among the collected samples were related to Merionespersicus (26.9\%), Tateraindica (25\%), Merioneshurrianae (24.1\%), Merioneslybicus (8.3\%) and Gerbillusnanus (15.7\%), respectively. Since the sampling was done in the burden of rural areas, no house mice (Mus Musculus) commonly reported in the studies for determination of rodent fauna, were caught.

So far, a few studies have been done related to broom tail rodents in Hormozgan Province. In the study conducted by Solymani et al. [14] only Rhombo-mysopimus species were collected from Kahorestan region in Bandar Abbas [14]. The remarkable point in the present study was that Rhombo-mysopimus species, the main reservoir host of the disease in most central areas and north eastern regions of Iran, did not collected. Although animal fauna may be different in various geographical conditions, the presence of high similarity of climatic conditions in Bastak and Kahorestan areas makes necessary revision and repeat of a similar study in Kahorestan area. Hanafi et al. [15] reported two species of Merionespersicus and Tateraindica in Haji Abad County. These two was also the dominant species in the current study. But no Leishmania contamination was observed by the researchers in the collected samples.

Leishmania contamination was observed with microscopic and molecular methods in the two rodent species of Merionespersicus and Tateraindica. Only one case of contamination to L. major was observed in Tateraindica species with both identification methods. One case of contamination in one female sample and two cases of contamination in two female samples were proved in Merioneshurrianae specie, using microscopic and specific PCR methods, respectively. It is worth noting that the microscopic method was only performed by investigation of prepared slides from rodents' ears. However, the molecular method was taken both on positive skin smears and the liver and spleen samples. So, the molecular method was able to identify positive the two slides prepared from ears which had been diagnosed negative by microscopic method. This reveals more sensitivity of the molecular method in detection of parasite.

Indian gerbil (Tateraindica) infected to L. major has been reported in several studies, especially in the west and the southwest regions of Iran. The species is reported by Javadian et al. [16] as the main reservoir host of wet Leishmaniasis in Ilam and Khozestan Provinces. Nesokiaindica and Merioneslibycus species are considered as the second reservoir hosts of the disease. The species were reported by Askari et al. [17] as Leishmania reservoir in Kherameh region, Fars Province. Contamination to the similar species was also detected using microscopic and molecular methods in Larestan County, Fars Province located in vicinity of Hormozgan Province. This was reported by Mehrabani et al. [18].

Indian desert jird species, Merioneshurrianae, is considered as the main rural reservoir host in the southeast of Iran, where Tateraindica acts usually as the second reservoir [19]. The infection of this species to L. major was identified by Mohebali et al. [20] using microscopic and RAPD-PCR methods and iso-enzymic analysis in the southeastern of Iran (e.g. the south of Baluchestan, 
and Dashtyari regions, Konarak and Chabahar). The contamination to this species has been limited in Iran to the southeastern areas and this contamination was also reported in the west of Hormozgan province in the present study. Merioneshurrianae species infected by L. major was also reported in Rajasthan Province in India [21].

Based on the extensive studies of researches, the contamination of Gerbillusnanus to L. major has not been mentioned in any scientific sources. For the first time and using microscopic and molecular methods, Mehrabani et al. [18] reported two rodent samples of Gerbillus spp. genus infected to L. major in Larestan region, Fars Province. They did not report the rodent species. However, based on the size of the samples (two cases of contaminations) which were big, the two samples were not certainly from $G$. nanus species. Of course, some cases of contamination to Gerbillus species, including Gerbilluspyramidum and Gerbillusdasyurus to L. major have been reported in some countries of Middle East, including Egypt, Jordan and Palestine. However, Gerbillusnanus was found in the present study but did not infect to L. major, parasite [22,23].

Two species of rodents' contaminations mentioned above were observed with both the microscopic and specific PCR methods. The molecular method was more successful in detection of contaminations, so that 2 and 3 cases of contaminations were identified using microscopic and molecular methods, respectively. Since parasite DNA is searched in PCR method and its sensitivity and specificity is high, can be expected that its' diagnostic power to be higher than the microscopic method which is highly dependent on skill and recognition ability of microscopist. This issue has been highlighted by studies of other researchers $[17,24]$.

\section{Conclusion}

Based on the above findings, leishmaniasis in this emerging focus was of rural or wet type and was caused by L. major. Two Merioneshurrianae and Tateraindica species of rodent were introduced as the disease reservoir hosts. So, considering the type of primers and used sequence in this study to differentiate species of cutaneous leishmaniasis pathogens in the city of Bastak, finding a practical method with high sensitivity and performance is essential to enhance accuracy and to provide possibility of early diagnosis

\section{References}

1. Nadim A, Javadian A, Mohebali M, Zameni M (2008) Leishmania parasite and Leishmaniasis. Center of Tehran University Press, Iran, pp. 20-32

2. Dedet JP, Pratlong F (2003) Leishmaniasis. In: Manson Cook PGC, Zulma A (Eds.), Manson's Tropical disease 21(4): 1339-1364.

3. Parvizi P, Moradi Gh, Amirkhani A (2009) Compare molecular methods with routine laboratory methods to detect leishmania parasite in animal reservoirs of rural cutaneous leishmaniasis. Journal of infectious and Tropical Diseases related to Tropical Pathology and Infectious Diseases Association 44: 13-15.

4. Aransay AM, Scoulica E, Tselentis Y (2000) Detection and Identification of Leishmania DNA within naturally infected sand flies by seminested pcr on minicircle kinetoplastic DNA. Appl Environ Microbiol 66(5): 1933-1938.

5. Faiman R, Abbasi I, Jaffe C, Motro Y, Nasereddin A, et al. (2013) A Newly emerged cutaneous leishmaniasis focus in northern Israel and two new reservoir hosts of Leishmania major. Neglected Tropical disease 7(2): 43-49.

6. Alvar J, Barker JR (2002) Molecular tools for epidemiological studies and diagnosis of leishmaniasis and selected other parasitic diseases. Trans $\mathrm{R}$ Soc Trop Med Hyg 96(Suppl 1): S1-S250.

7. Killick KR (1990) Phlebotomine vectors of the leishmaniases: a review. Med Vet Entomol 4(1): 1-24.

8. Killick KR, Ward RD (1981) Ecology of the Leishmania. Parasitology 82: 143-152.

9. Motazedian MH, Karamian M, Noyes HA, Ardehali S (2002) DNA extraction and amplification of Leishmania from archived, Giemsastained slides, for the diagnosis of cutaneous leishmaniasis by PCR. Ann Trop Med Parasitol 96(1): 31-34.

10. Rassi Y, Azizi K, Motazedian MH, Rafizadeh S, Fakhar M, et al. (2007) The seminested PCR based detection of Leishmania infantum infection in asymptomatic dogs in a new endemic focus of visceral leishmaniasis in Iran. Iranian J Arthropod Borne Dis 1(1): 38-42.

11. Azizi K, Rassi Y, Javadian E, Motazedian MH, Asgari Q et al. (2008) First detection of leishmania infantum in Phlebotomus (Larroussius) major (Diptera: Psychodidae) from Iran. J Med Entomol 45(4): 726-731.

12. Yaghoobi MR, Javadian E, Tahvildari GH (1994) The isolation of Leishmania major from Phlebotomus (Paraphlebotomus) caucasicus, in Isfahan province, Islamic Republic of Iran. Trans R Soc Trop Med Hyg 88(5): 518-519.

13. Azizi K, Rassi Y, Momenbellah MD (2010) PCR based detection of Leishmania major kDNA with in naturally infected Phlebotomuspapatasi in southern Iran. Trans Roy Soc Trop Med Hyg 104(6) : 440-442.

14. Soleimani AM, Javadian E, Reisi A, Yaghoobi MR (1998) Study on entomology fauna of psychodidae in Kahurestan area, Bandar Abbas. Hormozgan Medical Siences Journal 2: 25-30.

15. Hanafi AA, Yaghoobi MR, Zamani Q, Barzehkar A, Jaafari R, et al. (2006) Epidemiological aspects of cutaneous leishmaniasis in Hajiabad county, Hormozgan province 2003. Hormozgan Medical Siences Journal 10(1): $63-70$

16. Javadian E, Dehestani M, Nadim M, Rassi Y, Tahvildari BC, et al. (1998) Confirmation of Tateraindica (Rodentia: Gerbillidae) as the main reservoir host of zoonotic cutaneous leishmaniasis in the west of Iran. Iranian J Publ Health 27(1): 55-60.

17. Asgari Q, Motazedian MH, Mehrabani D, Oryan A, Hatam GR, et al. (2006) Zoonotic cutaneous Leishmaniasis in Shiraz, southern Iran: A molecular, isoenzyme and morphologic approach. Journal of Research in Medical Sciences 12(1): 7-15.

18. Mehrabani D, Motazedian MH, Oryan A, Asgari Q, Hatam GR, et al.(2007) A search for the hosts of Leishmania major in the Larestan region of southern Iran: demonstration of the parasite in Tateraindica and Gerbillus sp, by microscopy, culture and PCR. Ann Trop Med Parasitol 101(4): 315-322

19. Seyedi MA, Nadim A (1984) Cutaneous leishmaniosis in Baluchistan Iran. Abstract and Poster, International Congress for Tropical Medicine and Malaria. Calgari, Canada, USA, p.124.

20. Mohebali M, Javadian E, Yaghoobi MR, Akhavan AA, Hajjaran H, et al. (2004) Characterization of Leishmania infection in rodents from endemic areas of the Islamic republic of Iran. East Med Health J 10(4-5): 591- 599.

21. Morsy TA, Shoukry A, Schnur LF, Sulitzeanu A (1987) Gerbilluspyramidum is a host of Leishmnaia major in the Sinai peninsula. Ann Trop Med Parasitol 81: 741-742.

22. Peters W, Chance ML, Chowdhury AB, Ghosh Dastivar B, Nandy A, et al. (1981) The identity of some stocks of Leishmnaia isolated in India. Ann Trop Med Hyg 75(2): 247-249. 
23. Wesserberg G, Abramsky Z, Anders G, El Fari M, Schoenian G, et al. (2002) The ecology of cutaneous leishmaniasis in Nizzana, Israel: infection patterns in the reservoir host and epidemiological implications. Int J Parasitol 32(2): 133-143.
24. Alvar J, Barker JR (2002) Molecular tools for epidemiological studies and diagnosis of leishmaniasis and selected other parasitic diseases. Trans $\mathrm{R}$ Soc Trop Med Hyg 96: S243-250. (c) (i) Creative Commons Attribution 4.0

For possible submissions Click Here

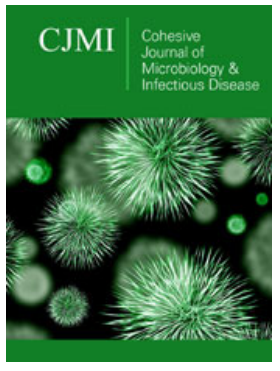

Cohesive Journal of Microbiology \& Infectious Disease

\section{Benefits of Publishing with us}

- High-level peer review and editorial services

- Freely accessible online immediately upon publication

- Authors retain the copyright to their work

- Licensing it under a Creative Commons license

- Visibility through different online platforms 